\title{
Triiodothyronine improves morphology and upregulates seladin-1 of neurospheres extracted from subventricular zone in streptozotocin- induced rat model of Alzheimer's disease
}

\author{
Tahmineh Mokhtari,a,b Simin Mahahakizadeh, ${ }^{c}$ Hadi Aligholi, ${ }^{d}$ Sahar ljaz, ${ }^{\text {ef }}$ Leila Noori, \\ Gholamreza Hassanzadeh ${ }^{\text {gh, }}, \mathrm{e}$
}

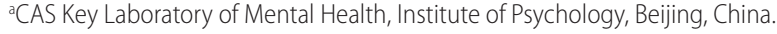 \\ bDepartment of Psychology, University of Chinese Academy of Sciences, Beijing, China. \\ cDepartment of Anatomy, School of Medicine, Alborz University of Medical Sciences, Karaj, Iran. \\ dDepartment of Neuroscience, School of Advanced Medical Sciences and Technologies, Shiraz University of Medical Sciences, Shiraz, Iran. \\ eDepartment of Anatomy, School of Medicine, Tehran University of Medical Sciences, Tehran, Iran. \\ fDepartment of Anatomy \& Histology, Faculty of Biosciences, University of Veterinary \& Animal Sciences, Lahore, Pakistan. \\ 9Department of Neuroscience and addiction studies, School of advanced technologies in medicine, Tehran University of Medical Sciences, Tehran, Iran. \\ hLegal medicine research center, Legal Medicine Organization, Tehran, Iran. \\ Correspondence to: Gholamreza Hassanzadeh (email: hassanzadeh@tums.ac.ir). \\ (Submitted: 21 November 2019 - Revised version received: 14 December 2019 - Accepted: 25 December 2020 - Published online: 26 February 2020)
}

\begin{abstract}
Objectives In this study, the effects of triiodothyronine (T3) on neurospheres isolated from subventricular zone (SVZ) of Alzheimer's disease (AD) induced rats were examined.

Methods Eighteen male Wistar rats were classified into two groups: Sham (Sh) and STZ (streptozotocin injected, $1.5 \mathrm{mg} / \mathrm{kg}$ in each lateral ventricle on days 1 and 3 after recovery). On day 21, the SVZ was extracted and neurospheres were cultured. T3 (50 nM) was added to the culture medium (STZ+T3 group) and then, the morphology and seladin-1 gene expression of neurospheres were evaluated.

Results The diameter and the number of neurospheres along with the gene expression of seladin- 1 were significantly decreased in the STZ group compared to Sh group $(P<0.05)$ while the administration of T3 significantly $(P<0.05)$ increased all these parameters in the STZ group.

Conclusion STZ decreases the proliferation of stem cells extracted from SVZ and administration of T3 to the culture media improves the morphology and upregulates the gene expression of seladin-1 of neurospheres.

Keywords Alzheimer's disease, subventricular zone, neurospheres, seladin-1.
\end{abstract}

\section{Introduction}

Alzheimer's disease (AD) is the most common progressive disorder in central nervous system (CNS) with effects on awareness contents and cognitive abilities. ${ }^{1,2}$ Alois Alzheimer introduced $\mathrm{AD}$ for the first time by dissecting the brain of a middle-aged woman who had suffered from memory deficits and lack of progressive abilities. ${ }^{1,3}$ Three obvious symptoms of $\mathrm{AD}$ are: (1) Atrophy of brain in which its weight drops, gyri narrow, sulci widen and ventricles dilate. (2) Acceleration of extracellular beta-amyloid plaques $(\mathrm{A} \beta$, also called neuritic or senile plaques) formed by the amyloid precursor protein (APP) assembling. (3) Neurofibrillary tangles attributed neuronal death: Abnormalities in the cellular skeleton upset the axonal transport due to disorganization of microtubules involved there-in. ${ }^{4}$ In terms of prevalence, $1 / 8$ th or, in other words, about $10 \%$ of people over the age of 65 are afflicted with AD. ${ }^{5}$ $\mathrm{AD}$ is divided into two main types: (1) Familial Alzheimer's disease (FAD) or the inherited type that occurs prematurely and (2) Sporadic Alzheimer disease (SAD) that occurs individually without genetic mutations, often in the 80 's. ${ }^{5}$

In this study, intracerebroventricular (ICV) injection of streptozotocin (STZ), as a diabetogenic factor, was used to suppress glucose metabolism. The STZ disrupts the regulating role of insulin and its receptor resulting in destroyed glucose metabolism in brain, lack of energy, lack of cholinergic system, and nerve growth along with learning, memory, general, and local anomalies. ${ }^{6}$ All of these disorders are attributed to STZ that mediates glucose metabolism by means of insulin signaling cascade. ${ }^{4,7}$

To induce progressive deterioration, like the one seen in SAD type, STZ is injected which causes a significant reduction of energy in hippocampal and cerebral cortical tissue and therefore, a disruption in the energy associated processes including eating, sorting, and packing of proteins in the Golgi organelles. The transmission of neurotransmitters, learning behaviors, memory, and energy-rich compounds are progressively affected. ${ }^{8}$ The progressive decline in short- and longterm memory in the post-administration phase of intravenous injection and the resultant disorders in behavior and glucose metabolism make STZ suitable for SAD model. Morris water maze (MVM) and passive avoidance memory behavioral tests have confirmed that $\mathrm{STZ}$ produces $\mathrm{AD}{ }^{9}$

It was believed for many years that the CNS has no neuroregenerative ability at adulthood. Nearly a century ago, as the technology progressed, cells that can replicate and differentiate into neurons and glia were identified in some areas of the brain and spinal cord. And until now, active neuronal activity in the subventricular zone (SVZ) in lateral wall of lateral ventricles, Subgranular zone of dentate gyrus, and hypothalamic subependymal zone has been documented. ${ }^{10-12}$ In the early part of the century, Allen identified mitotic cells in the SVZ of lateral ventricles in mature rats. Ventricular region forms during embryonic development near the 
ventricular area and is very noticeable in the ganglionprotuberance region. ${ }^{13}$ It is composed of a number of cells, possibly, B1 cells, which are the same as astrocytes. They are stem cells in the ventricular region, and these cells divide and make type C-cells that are capable of rapid proliferation. C-type cells, in turn, increase and convert to type A cells that are migratory neuroblasts. A-cells are surrounded by cells of type B1 and C. ${ }^{14,15}$

Preconditioning of stem cells with growth factors can improve their ability of proliferation and development and also promotes neuronal regeneration and survival. ${ }^{16}$ Among these factors, thyroid hormones (THs) such as triiodothyronine (T3) are critical for brain development. ${ }^{17}$ Although, T3 is less than thyroxine [T4], it is the most active form of THs. ${ }^{18}$ Furthermore, the neuroprotective impacts of THs are related to enhancement of neurotrophic factors (NFs) and their anti-apoptotic and anti-inflammatory mechanisms have been proven. ${ }^{19}$

In this study, we decided to evaluate the effects of this hormone on the level of seladin-1 expression in the neurospheres isolated from SVZ in $\mathrm{AD}$-induced rats.

\section{Methods and Materials}

\section{Study Design and Animals}

A total number of 18 male Wistar albino rats (100-150 g) were purchased from Shefa Neuroscience Research Center, Khatam Alanbia Hospital, Tehran, Iran. All animals were maintained in a clean and hygienic environment, on a $12-\mathrm{h}$ light and dark cycle and $23 \pm 2{ }^{\circ} \mathrm{C}$ temperature, and had access to food and water ad libitum. All procedures were carried out in accordance with the guidelines of the Iranian Council for use and care of animals and approved by Ethical Committee of Tehran University of Medical Sciences. This study was granted by Iran National Science Foundation (Grant No. 93009595). Subjects were randomly divided into two different groups: sham (Sh) group which received $5 \mathrm{~mL}$ normal saline in each lateral ventricle, STZ group which received $1.5 \mathrm{mg} / \mathrm{kg} \mathrm{STZ}$ in $5 \mathrm{~mL}$ normal saline in each lateral ventricle on days 1 and 3 . All subjects were housed in keeping cages in the resting time.

\section{Stereotaxic Surgery}

Rats were deeply anesthetized with ketamine $(100 \mathrm{mg} / \mathrm{kg})$ and xylazine (25 mg/kg) (both Razi Co., Iran) intraperitoneally. For chronic implantation of cannula (27-Gauge) into lateral cerebral ventricles (ICV) under stereotactic guidance, appropriate narcosis was verified by reflex testing such as the lack of ocular reflex and the absence of a pedal withdrawal response to a hard pinch. Therefore, animals were fixed in a stereotactic apparatus, and a midline incision was made on the cranial skin. Then, a small hole was induced in the cranial region and the guide cannula was implanted and fixed. Coordinates were AP-0.8 $\mathrm{mm}, \mathrm{L} \pm 1.5 \mathrm{~mm}$ (midline) and $3.6 \mathrm{~mm}$ deep from the dura mater. Animals were allowed 1 day to recover before STZ injection. STZ and its vehicle were administered on days 1 and 3 after recovery with a $10 \mu \mathrm{L}$ Hamilton syringe ( $5 \mu \mathrm{L}$ in each ventricle at a rate of $1 \mu \mathrm{L} / \mathrm{min}$ ) connected via a Teflon tube to an injector that exceeded by $2 \mathrm{~mm}$ the length of the guide cannula. ${ }^{4,7}$

\section{Morris Water Maze}

At the end of the experiment, to confirm the defects in learning and memory of animals, their behaviors were assessed by MWM test based on the previously described methods. ${ }^{20}$ It consisted of a circular water tank $(160 \mathrm{~cm}$ diameter, $60 \mathrm{~cm}$ height) filled with water $\left(25 \pm 1{ }^{\circ} \mathrm{C}\right)$ to a depth of $25 \mathrm{~cm}$. A non-toxic water dispersible emulsion was used to render the water opaque. Four equally spaced locations around the edge of the pool (North, South, East, and West) were used as start points which divided the pool into four quadrants. An escape platform $(10 \mathrm{~cm}$ in diameter) was placed in the pool $2 \mathrm{~cm}$ below the surface of water. The escape platform was placed in the middle of one of the randomly selected quadrants of the pool and kept in the same position throughout the entire experiment (north-east for this study). Animals received a training session consisting of four trials per session (once from each starting point) for 4 days (days 16, 17, 18, and 19), each trial having a ceiling time of $90 \mathrm{~s}$ and a trial interval of approximately $30 \mathrm{~s}$. After climbing onto the hidden platform, animals remained there for $30 \mathrm{~s}$ before commencement of the next trial. If the rat failed to locate the hidden platform within the maximum time of $90 \mathrm{~s}$, it was gently placed on the platform and allowed to remain there for the same interval of time. The time taken to locate the hidden platform (latency in s) was measured. Twenty-four hours after the acquisition phase, a probe test (day 20) was conducted by removing the platform. Rats were allowed to swim freely in the pool for $90 \mathrm{~s}$ and the time spent in target quadrant, which had previously contained the hidden platform, was recorded. The time and distance spent in the target quadrant indicated the degree of memory consolidation which had taken place after learning.

\section{Cell Culture}

On day 21, animals were sacrificed from both the groups (Sh and STZ) and the SVZ was isolated. The tissue samples were digested after the mechanical digestion with $250 \mu \mathrm{L}$ trypsin $0.05 \%$ for $4 \mathrm{~min}$ and centrifuged by $810 \mathrm{rpm}$ for $5 \mathrm{~min}$ after adding trypsin inhibitor. Cell deposition in neurosphere cell culture, that was consist of DMEM + F12 (gibco) glutamine $1 \%$ (gibco), B27 supplement 1\% (gibco) and epidermal growth factor $20 \mathrm{ng} / \mathrm{mL}$ (sigma), was cultivated in incubator with $37{ }^{\circ} \mathrm{C}$ temperature, 95\% humidity, and $5 \% \mathrm{CO}_{2}$. Within 15 days, nerve/protozoal stem cells were proliferated as a series of spherical cells called neurospheres. By increasing the volume of the neurospheres, the cell passage by collecting the neurons in the Falcon tube and centrifuging for $5 \mathrm{~min}$ at $810 \mathrm{rpm}$ was performed. To test the effects of T3 on neurospheres, T3 $(50 \mathrm{nM})$ was added to the medium (STZ + T3 group). Medium was renewed every 2-3 days.

\section{Morphometric Studies}

In order to evaluate cell proliferation, both the numbers and diameters of neurospheres were considered. On the seventh day of cultivation, five microscopic images were taken randomly from each container. Cell counting was performed by using Trypan blue and Neobar lam and after the second passage, the diameter and number of neurons were measured. Number of neurospheres was counted by infinity software. The neurospheres diameter were measured by using the infinity software 
and the results were described as the mean of two perpendicular diameters to the diameter of the neurospheres. The number and diameter of the neurospheres and cells were compared.

\section{Real-Time PCR}

Expression of Seladin-1 in neurospheres was measured by Real-time PCR. Total RNA was isolated from hemispheres, then mRNA $(1 \mu \mathrm{g})$ was converted to cDNA via reverse transcription using First Strand cDNA Synthesis Kit (Thermo Scientific, USA). Specific primers along with cDNA and PCR reagents (polymerase, dNTP, magnesium and buffer; $5 \times$ HOT FIREPol $^{\oplus}$ EvaGreen $^{\circledR}$ qPCR Mix Plus [ROX] $1 \mathrm{~mL}$, Solis Bio Dyne, Estonia) were placed on the three-color real-time PCR machine (Applied Biosystems Step One, USA) for further analysis. At first, incubation of samples was performed at $95^{\circ} \mathrm{C}$ for $15 \mathrm{~min}$ for initial polymerase activation. Then, samples underwent the three subsequent phases as follows: (1) denaturation (at $95{ }^{\circ} \mathrm{C}$ for $15 \mathrm{~s}$ ); (2) annealing (at $60{ }^{\circ} \mathrm{C}$ for $20 \mathrm{~s}$ ); and (3) elongation (at $72^{\circ} \mathrm{C}$ for $20 \mathrm{~s}$ ). Finally, quantification of data, its normalization to GAPDH and fold change comparison to the control were performed. ${ }^{21}$ In Table 1, the nucleotide sequences of primers are shown.

\begin{tabular}{lll}
\hline Table 1. & & \\
\hline Primer & & Sequence \\
\hline \multirow{2}{*}{ Seladin-1 } & Forward & 5'-GGGTGTTTGTGTGCCTCTTCC-3' \\
& Reverse & 5'-GCTCCTTCCACTCCCGTACC-3' \\
& Forward & '-CTGTCTGGCGGCACCACCAT-3' \\
b-actin & Reverse & 5'-GCAACTAAGTCATAGTCCGC-3' \\
& & \\
\hline
\end{tabular}

\section{Statistical analysis}

All data were analyzed through one-way analysis of variance (ANOVA) and the post-hoc Tukey's, and two-way ANOVA and the Bonferroni's multiple comparison tests were applied for behavioral data belonging to learning and memory skills using Graph-Pad PRISM version 6 Software (San Diego, California, USA). Results were represented as mean \pm SEM and $\mathrm{p}<0.05$ was considered significant.

\section{Results \\ Effects of ICV injection of STZ on learning and memory in the rat model of $A D$}

Injection of STZ induced defect in spatial learning and memory of STZ group. According to Fig. 1, the mean latency time to reach the hidden platform $(\mathrm{P}<0.05$, Fig. 1a) and distance traveled in the MVM $(\mathrm{P}<0.05$, Fig. $1 \mathrm{~b})$ were significantly increased in STZ group compared to Sh group. Moreover, STZ-treated groups spent significantly less time $(\mathrm{P}<0.05$, Fig. 1c) and distances in the platform quadrant $(P<0.05$, Fig. $1 \mathrm{~d})$ compared to Sh group.

\section{Effects of T3 on the number of neurospheres extracted from SVZ in the rat model of $A D$}

The number of neurospheres decreased in the AD model of rat compared with Sh group $(\mathrm{p}<0.05$, Fig. 1). Culturing with medium contained T3 increased the number of neurospheres in the STZ + T3 neurospheres compared to STZ group $(\mathrm{p}<0.05$, Fig. 1).

\section{Effects of T3 on diameter of neurospheres extracted from SVZ in the rat model of AD}

Based on the results, the diameter of neurospheres decreased in the $\mathrm{AD}$ model group (STZ group) compared with Sh group ( $p<0.05$, Fig. 2 ). The diameter of neurospheres in the STZ + T3 increased compared to STZ group $(p<0.05$, Fig. 1$)$.

\section{Effects of T3 on seladin-1 gene expression of neurospheres extracted from SVZ in the rat model of $A D$}

Based on the results, the seladin-1 gene expression decreased in the AD model group (STZ group) compared to Sh group ( $\mathrm{p}<0.05$, Fig. 2). The seladin-1 gene expression in T3 group increased compared to STZ group ( $<<0.05$, Fig. 1).

\section{Discussion}

In this study, the effects of T3 on morphology and seladin-1 gene expression of neurospheres extracted from SVZ were investigated in rat model of AD. In this study, STZ was used to induce $\mathrm{AD}$ in animals. $\mathrm{AD}$ is the most common cause of dementia diagnosed after the age of 60 , and it is characterized by neuronal loss as a consequence of neurofibrillary tangles and senile plaques. There are different theories which explain the accumulation of beta amyloid plaques, but abnormal levels of oxidative stress have been reported in both brain and blood stream in $\mathrm{AD}^{22,23}$ An enhanced oxidative stress induced modified proteins and mitochondrial dysfunction in $\mathrm{AD}$ brain have been reported, ${ }^{24,25}$ and it has also been suggested that oxidative stress is a key for the progression of $\mathrm{AD} .{ }^{26}$ The mechanism of $\mathrm{AD}$ development in the brain is unknown. However, different studies have proved that chronic ICV injections of STZ can reduce uptake of cerebral glucose and induce multiple other effects that resemble behavioral, molecular, pathological characteristics of $\mathrm{AD}{ }^{27}$

The results of present study revealed that the diameter and the number of neurospheres were significantly decreased in the STZ treatment group compared to Sh group.

$\mathrm{AD}$, the widespread cause of dementia, is characterized by progressive neurodegeneration and the appearance of specific histopathological markers represented by focal extracellular deposits of fibrillar $A \beta$ in the brain parenchyma and in the wall of blood vessels, and by the intraneuronal accumulation of neurofibrillary tangles formed as a result of the abnormal hyperphosphorylation of cytoskeletal Tau filaments. ${ }^{28}$ The initial neurodegenerative events of $\mathrm{AD}$ appear in the transentorhinal cortex, and subsequently spread to the entorhinal cortex and to the hippocampus. ${ }^{29,30} \mathrm{SVZ}$, as a neurogenic niche, contains multipotent neural stem cells (NSCs). ${ }^{31,32}$ The NSCs that exhibit slow self-renewal produce neural progenitor cells with a faster dividing cell cycle, which ultimately differentiate into neurons or neuroglia; the differentiation process is regulated by numerous trophic factors. ${ }^{33,34}$ The majority of studies performed on transgenic animals expressing the mutant APP demonstrated decreased neurogenesis either in the Dentate Gyrus (DG) or in both the DG and the SVZ. ${ }^{35-38}$ In a previous similar study, Mozhdeh et al. demonstrated that the ICV 

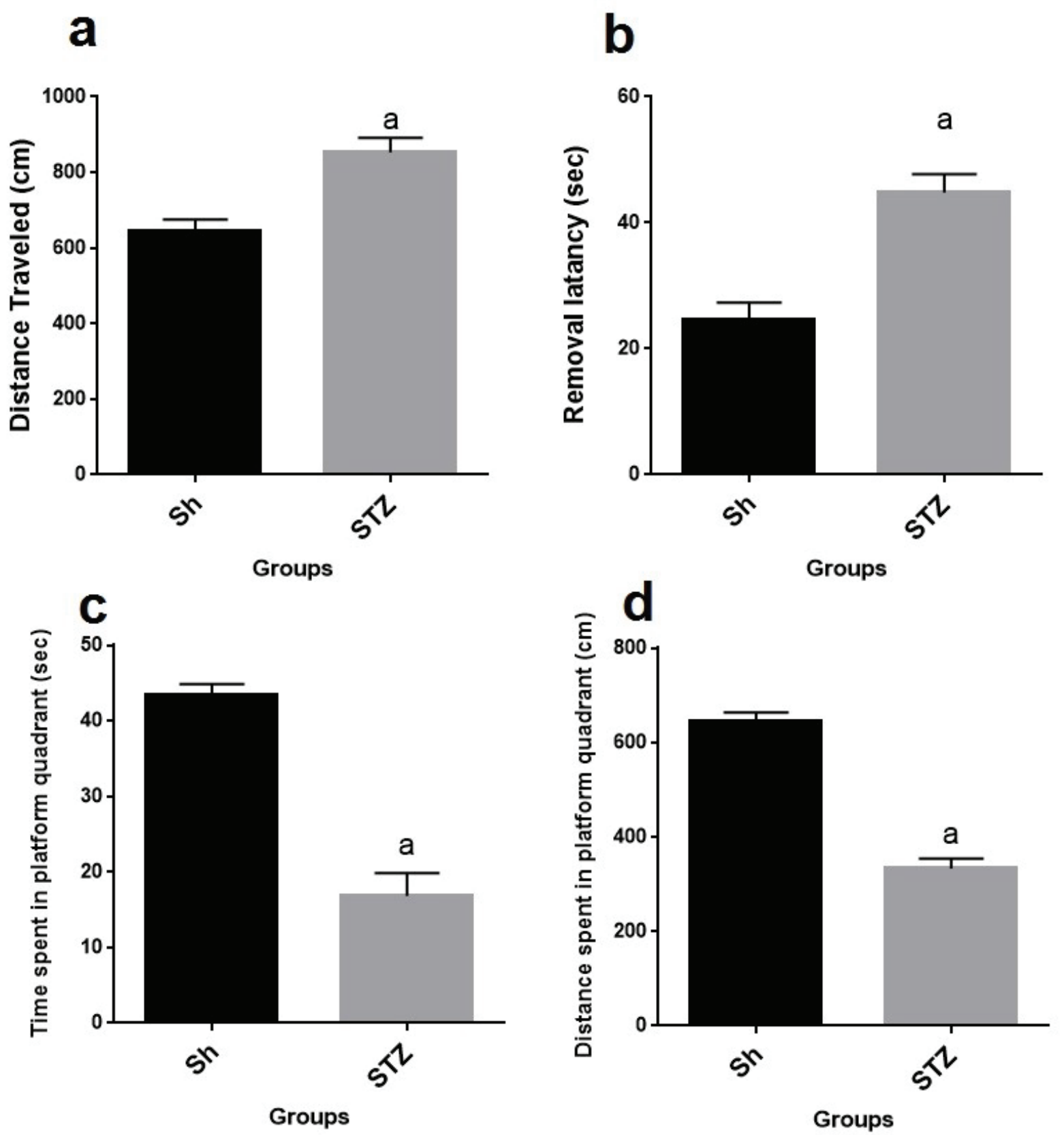

Fig. 1 Effects of ICV injection of STZ on spatial memory and learning in the rat model of AD. (a) Latency to platform (s), (b) sistance traveled to platform, (c) time, and (d) distance spent in the platform quarter. $\mathrm{p}<0.05$ compared to Sh group. Sh: sham group with ICV injection of normal saline; STZ: animals with ICV injection of STZ

injection of STZ impaired the proliferation feature of SVZ extracted neurospheres in a rat model of $\mathrm{AD}^{9}$ which confirmed the results of present study.

The results of present study showed that T3 could increase the seladin-1 gene expression in the stem cells extracted from SVZ in the rat model of AD.

The mechanisms of TH levels alteration as a factor to increase the mobility towards $\mathrm{AD}$ in older people is unclear. Numerous researches tried to find a relation between AD and TH levels. In a study by Ceresini et al., ${ }^{39} 1171$ participants from Italy were analyzed and it was reported that dysfunction of thyroid gland tends to be more frequent in older people than in youngsters, with subclinical hyperthyroidism being the most prevalent condition. Their findings demonstrated an independent association between cognitive impairment and subclinical hyperthyroidism. Their findings were similar to those obtained from Kalmijn et al. investigation. ${ }^{40}$

Some studies have demonstrated a very high prevalence of autoimmune thyroid disease in familial AD. ${ }^{41,42}$ Similarly, Ganguli et al. reported that the subclinical hypothyroidism state correlates with cognitive disorders in patients aged $\geq 65 .{ }^{43}$

An alternative mechanism of $\mathrm{TH}$ to improve $\mathrm{AD}$ has been described. The increase of the seladin-1 gene and protein expression of seladin-1 (selective AD indicator 1) gene cause decrease neuronal death, ${ }^{31,44}$ promotes cholesterol synthesis inside the neuron, ${ }^{45}$ and reduce $\mathrm{A} \beta$ accumulation by inhibition 
Sh

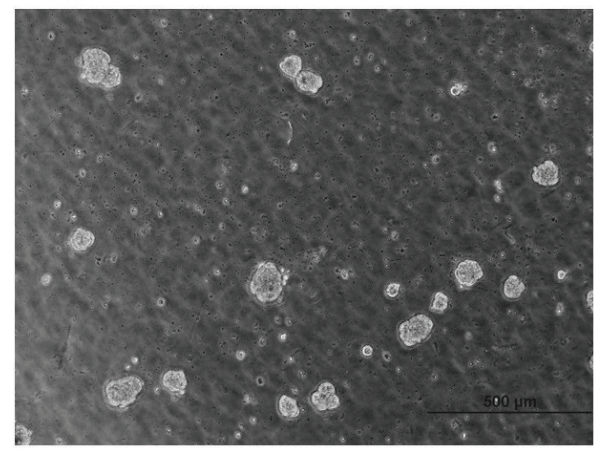

STZ+T3

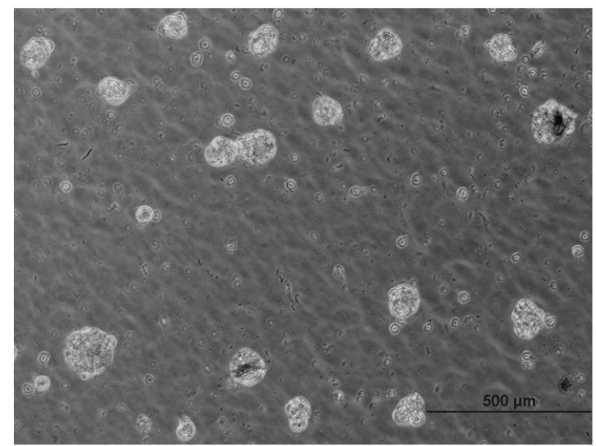

STZ
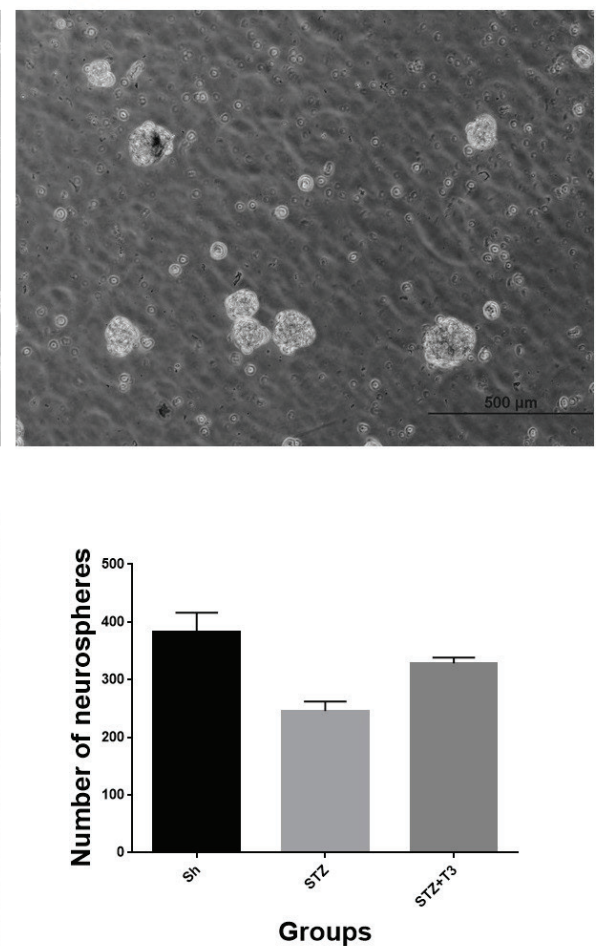

Fig. 2 Effects of T3 on the number of neurospheres extracted from SVZ in the rat model of AD. (a) $p<0.05$ compared to Sh group,

(b) $p<0.05$ compared to STZ group. Sh: neurospheres isolated from rats received ICV injection of normal saline; STZ: neurospheres isolated from rats received ICV injection of STZ, STZ + T3: neurospheres isolated from rats received ICV injection of STZ and cultured with T3.

Sh

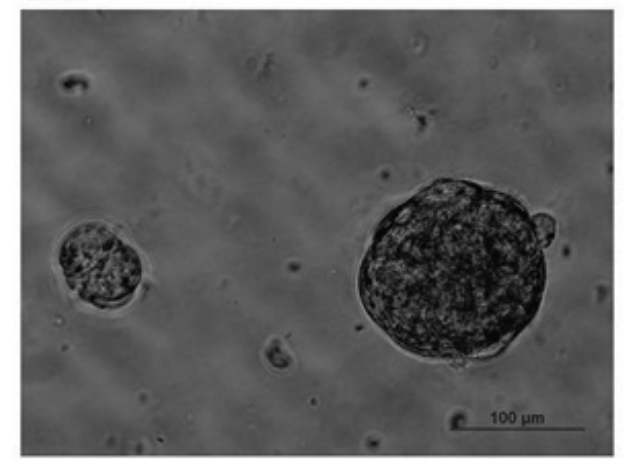

\section{STZ+T3}

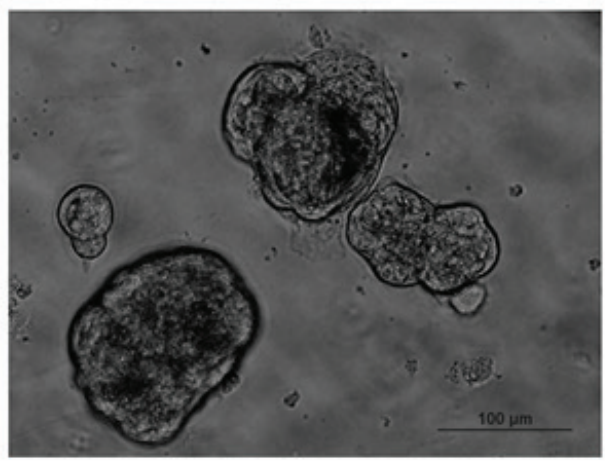

STZ
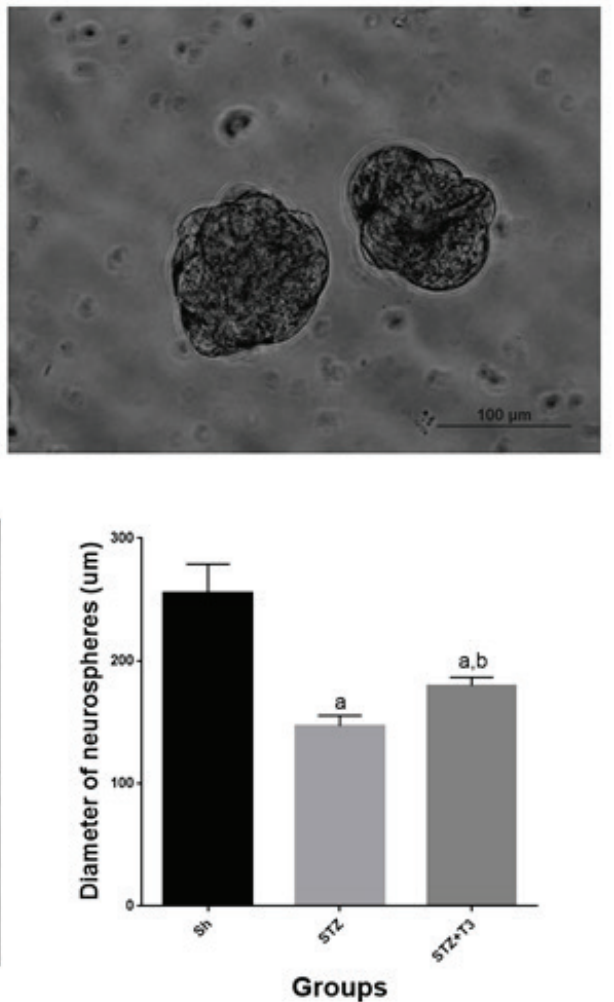

Fig. 3 Effects of T3 on seladin-1 gene expression of neurospheres extracted from SVZ in the rat model of AD. The gene expression of seladin-1 was investigated using real-time PCR. (a) $p<0.05$ compared to Sh group, (b) $p<0.05$ compared to STZ group. Sh: neurospheres isolated from rats received ICV injection of normal saline; STZ: neurospheres isolated from rats received ICV injection of STZ; STZ + T3: neurospheres isolated from rats received ICV injection of STZ and cultured with T3. 


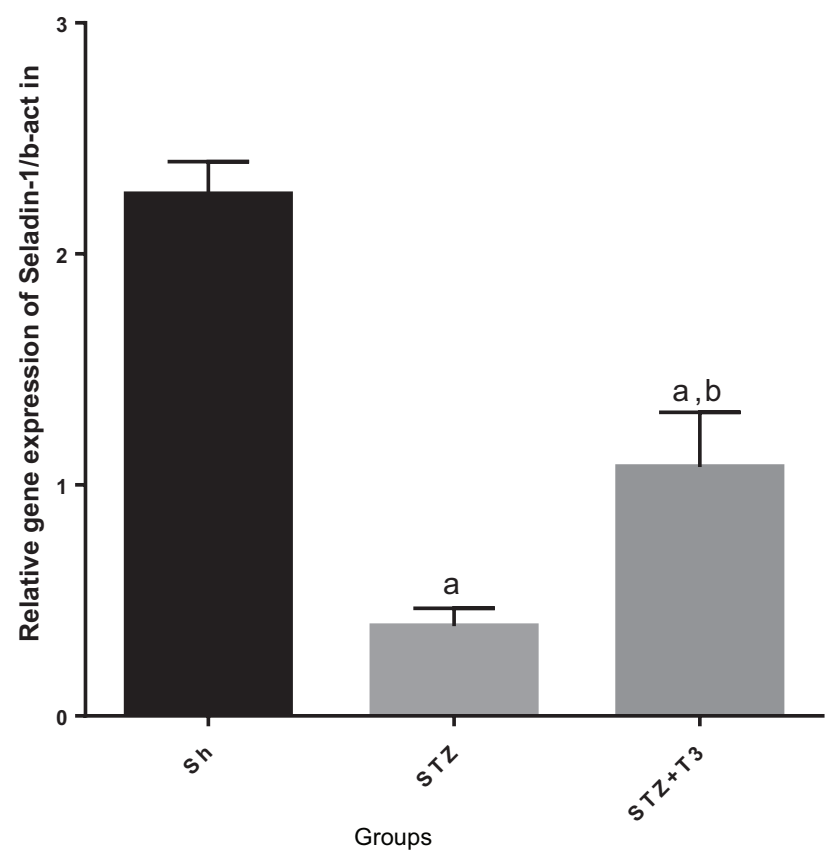

Fig. 3 Effects of T3 on the diameter of neurospheres extracted from SVZ in the rat model of AD. (a) $p<0.05$ compared to Sh group, (b) $p<0.05$ compared to STZ group. Sh: neurospheres isolated from rats received ICV injection of normal saline; STZ: neurospheres isolated from rats received ICV injection of STZ; STZ +T3: neurospheres isolated from rats received ICV injection of STZ and cultured with T3. of co-localization of $\mathrm{A} \beta$ precursor protein. ${ }^{46} \mathrm{TH}$ exerts an essential effect on the maintenance and development of CNS. ${ }^{47}$

The effectiveness of $\mathrm{TH}$ on seladin-1 gene expression was investigated. Ishida et al. reported that $\mathrm{TH}$ upregulated the expression of seladin-1, a gene related to AD in whole cell extract from mouse forebrain. ${ }^{44}$ Benvenuti et al. reported that TH increased TR- $\alpha 1$, TR- $\beta 1$, and TR- $\beta 2$ and seladin- 1 gene expression in an in vitro model of human neuronal precursor cell lines. ${ }^{48}$ Likewise, the beneficial effects of T3 on neurodegenerative diseases have been presented in a study by Mokhtari et al., it was proven that injection of $\mathrm{T} 3$ improved memory and learning by upregulation of brain-derived neurotropic factor and Glial cell-derived neurotropic factor in CA1 hippocampal region in rat model of ischemic brain stroke. ${ }^{49}$

\section{Conclusion}

The results obtained from this study showed that the ICV injection of STZ decreases the number and diameter of neurospheres and seladin-1 gene expression in rats (model of AD). Additionally, adding T3 to the culture medium of these neurospheres improves their morphological characteristics and increases seladin-1 gene expression.

\section{Acknowledgement}

This work was funded by the Iran National Science Foundation (Grant 93009595).

\section{References}

1. Katzman R. The prevalence and malignancy of Alzheimer disease: A major killer. Arch. Neurol. 1976;33(4):217-8.

2. Hassanzadeh G, Hosseini A, Pasbakhsh P, Akbari M, Ghaffarpour M, Takzare $\mathrm{N}$, et al. Trimetazidine prevents oxidative changes induced in a rat model of sporadic type of Alzheimer's disease. Acta Med. Iran. 2015;53(1):17-24.

3. Kumar A, Singh A. A review on Alzheimer's disease pathophysiology and its management: an update. Pharmacol. Rep. 2015;67(2):195-203.

4. Hassanzadeh G, Fallahi Z, Khanmohammadi M, Elmizadeh H, Sharifzadeh M, Nouri K, et al. Effect of magnetic tacrine-loaded chitosan nanoparticles on spatial learning, memory, amyloid precursor protein and seladin-1 expression in the hippocampus of streptozotocin-exposed rats. Int. Clin. Neurosci. J. 2016;3(1):25-31.

5. Peri A, Serio M. Neuroprotective effects of the Alzheimer's disease-related gene seladin-1. J. Mol. Endocrinol. 2008;41(5):251-61.

6. Condello C, Lemmin T, Stöhr J, Nick M, Wu Y, Maxwell AM, et al. Structural heterogeneity and intersubject variability of $A \beta$ in familial and sporadic Alzheimer's disease. Proc. Natl Acad. Sci. 2018:201714966.

7. Biasibetti R, dos Santos JPA, Rodrigues L, Wartchow KM, Suardi LZ, Nardin P, et al. Hippocampal changes in STZ-model of Alzheimer's disease are dependent on sex. Behav. Brain Res. 2017;316:205-14.

8. Guigon CJ, Zhao L, Lu C, Willingham MC, Cheng S-y. Regulation of $\beta$-catenin by a novel nongenomic action of thyroid hormone $\beta$ receptor. Mol. Cell. Biol. 2008;28(14):4598-608.

9. Mozhdeh HP, Zeynali B, Aligholi H, Radgerdi IK, Negah SS, Hassanzadeh G. The effect of intracerebroventricular administration of streptozocin on cell proliferation in subventricular zone stem cells in a rat model of alzheimer's disease. Neurosci. J. Shefaye Khatam. 2015;3:80-6.

10. Andreotti JP, Lousado L, Magno LAV, Birbrair A. Hypothalamic neurons take center stage in the neural stem cell niche. Cell Stem Cell. 2017;21(3):293-4.

11. Shan X, Tomlinson L, Yang Q, Colognato H. Distinct requirements for extracellular and intracellular MMP12 in the development of the adult V-SVZ neural stem cell niche. Stem Cell Rep. 2018;10(3):984-99.

12. Corsini NS, Knoblich JA. Tracing stem cell division in adult neurogenesis. Cell Stem Cell. 2018;22(2):143-5.

13. Aligholi H, Hassanzadeh G, Azari H, Rezayat SM, Mehr SE, Akbari M, et al. A new and safe method for stereotactically harvesting neural stem/

progenitor cells from the adult rat subventricular zone. J. Neurosci.
2014;225:81-9.
Subventricular zone astrocytes are neural stem cells in the adult
Doetsch F, Caille I, Lim DA, García-Verdugo JM, Alvarez-Buylla A. Subventricular zone astrocytes are neural stem
mammalian brain. Cell. 1999;97(6):703-16.

15. Fuentealba LC, Obernier K, Alvarez-Buylla A. Adult neural stem cells bridge their niche. Cell Stem Cell. 2012;10(6):698-708.

16. Xiong $L-L$, Chen Z-W, Wang T-H. Nerve growth factor promotes in vitro proliferation of neural stem cells from tree shrews. Neural Regen. Res. 2016;11(4):591-6.

17. De Escobar GM, Obregón MJ, Del Rey FE. Role of thyroid hormone during early brain development. Eur. J. Endocrinol. 2004;151(Suppl 3):U25-U37.

18. Yen PM. Physiological and molecular basis of thyroid hormone action. Physiol. Rev. 2001;81(3):1097-142.

19. Genovese T, Impellizzeri D, Ahmad A, Cornelius C, Campolo M, Cuzzocrea $S$, et al. Post-ischaemic thyroid hormone treatment in a rat model of acute stroke. Brain Res. 2013;1513:92-102.

20. Mahakizadeh S, Akbari M, Sharifzadeh M, Rastegar T, Abolhassani F, Hassanzadeh G. Wharton'jelly mesenchymal stem cells and insulin effect on BDNF expression in CA1 and CA3 regions of rats' hippocampus after chronic hypoxia. J. Contemp. Med. Sci. 2018;4(2):63-9.

21. Sabbaghziarani F, Mortezaee K, Akbari M, Soleimani M, Moini A, Ataeinejad $\mathrm{N}$, et al. Retinoic acid-pretreated Wharton's jelly mesenchymal stem cells in combination with triiodothyronine improve expression of neurotrophic factors in the subventricular zone of the rat ischemic brain injury. Metab. Brain Dis. 2017;32(1):185-93.

22. Sutherland GT, Chami B, Youssef P, Witting PK. Oxidative stress in Alzheimer's disease: Primary villain or physiological by-product? Redox Rep. 2013;18(4):134-41.

23. Schrag M, Mueller C, Zabel M, Crofton A, Kirsch W, Ghribi O, et al. Oxidative stress in blood in Alzheimer's disease and mild cognitive impairment: a meta-analysis. Neurobiol. Dis. 2013;59:100-10.

24. Butterfield DA, Reed T, Newman SF, Sultana R. Roles of amyloid $\beta$-peptideassociated oxidative stress and brain protein modifications in the pathogenesis of Alzheimer's disease and mild cognitive impairment. Free Radic. Biol. Med. 2007;43(5):658-77. 
25. Beal MF. Mitochondrial dysfunction and oxidative damage in Alzheimer's and Parkinson's diseases and coenzyme Q 10 as a potential treatment. J. Bioenerg. Biomembr. 2004;36(4):381-6.

26. Sultana R, Butterfield DA. Role of oxidative stress in the progression of Alzheimer's disease. J. Alzheimer's Dis. 2010;19(1):341-53.

27. Grieb P. Intracerebroventricular streptozotocin injections as a model of Alzheimer's disease: In search of a relevant mechanism. Mol. Neurobiol. 2016;53(3):1741-52.

28. Selkoe DJ. Alzheimer's disease: genes, proteins, and therapy. Physiol. Rev. 2001;81(2):741-66

29. Thompson PM, Hayashi KM, Dutton RA, Chiang MC, Leow AD, Sowell ER, et al. Tracking Alzheimer's disease. Ann. NY Acad. Sci. 2007;1097(1):183-214

30. Thompson PM, Hayashi KM, De Zubicaray G, Janke AL, Rose SE, Semple $J$, et al. Dynamics of gray matter loss in Alzheimer's disease. J. Neurosci. 2003;23(3):994-1005.

31. Al-Mahmood S, Colin S, Schneider C. Composition comprising an antisense sequence implicated in the regulation of angiogenesis. Google Patents; 2012

32. Taupin $\mathrm{P}$, Gage FH. Adult neurogenesis and neural stem cells of the central nervous system in mammals. J. Neurosci. Res. 2002;69(6):745-9.

33. Seaberg RM, Van Der Kooy D. Adult rodent neurogenic regions: The ventricular subependyma contains neural stem cells, but the dentate gyrus contains restricted progenitors. J. Neurosci. 2002;22(5):1784-93.

34. Abrous DN, Koehl M, Le Moal M. Adult neurogenesis: from precursors to network and physiology. Physiol. Rev. 2005;85(2):523-69.

35. Feng R, Rampon C, Tang Y-P, Shrom D, Jin J, Kyin M, et al. Deficient neurogenesis in forebrain-specific presenilin-1 knockout mice is associated with reduced clearance of hippocampal memory traces. Neuron. 2001:32(5):911-26.

36. Haughey NJ, Nath A, Chan SL, Borchard A, Rao MS, Mattson MP. Disruption of neurogenesis by amyloid $\beta$-peptide, and perturbed neural progenitor cell homeostasis, in models of Alzheimer's disease. J. Neurochem. 2002;83(6):1509-24.

37. Wang R, Dineley K, Sweatt J, Zheng H. Presenilin 1 familial Alzheimer's disease mutation leads to defective associative learning and impaired adult neurogenesis. Neuroscience. 2004;126(2):305-12.

38. Zhang C, McNeil E, Dressler L, Siman R. Long-lasting impairment in hippocampal neurogenesis associated with amyloid deposition in knock-in mouse model of familial Alzheimer's disease. Exp. Neurol. 2007:204(1):77-87.
39. Ceresini G, Lauretani F, Maggio M, Ceda GP, Morganti S, Usberti E, et al Thyroid function abnormalities and cognitive impairment in elderly people: results of the Invecchiare in Chianti study. J. Am. Geriat. Soc. 2009;57(1):8993.

40. Kalmijn S, Mehta KM, Pols HA, Hofman A, Drexhage HA, Breteler MM. Subclinical hyperthyroidism and the risk of dementia. The Rotterdam study. Clin. Endocrinol. 2000;53(6):733-7.

41. Lopez OL, Rabin BS, Huff FJ, Rezek D, Reinmuth OM. Serum autoantibodies in patients with Alzheimer's disease and vascular dementia and in nondemented control subjects. Stroke. 1992;23(8):1078-83.

42. Ewins DL, Rossor MN, Butler J, Rogues PK, Mullen MJ, McGregor AM Association between autoimmune thyroid disease and Familial Alzheimers disease. Clin. Endocrinol. 1991:35(1):93-6.

43. Ganguli M, Burmeister LA, Seaberg EC, Belle S, DeKosky ST. Association between dementia and elevated TSH: A community-based study. Biol. Psychiatry. 1996;40(8):714-25.

44. Ishida E, Hashimoto K, Okada S, Satoh T, Yamada M, Mori M. Crosstalk between thyroid hormone receptor and liver $X$ receptor in the regulation of selective Alzheimer's disease indicator-1 gene expression. PLoS One. 2013:8(1):e54901.

45. Waterham HR, Koster J, Romeijn GJ, Hennekam RC, Vreken P, Andersson $\mathrm{HC}$, et al. Mutations in the 3beta-hydroxysterol Delta24-reductase gene cause desmosterolosis, an autosomal recessive disorder of cholesterol biosynthesis. Am. J. Human Genet. 2001;69(4):685-94.

46. Greeve I, Hermans-Borgmeyer I, Brellinger C, Kasper D, Gomez-Isla T, Behl C, et al. The human DIMINUTO/DWARF1 homolog seladin-1 confers resistance to Alzheimer's disease-associated neurodegeneration and oxidative stress. J. Neurosci. 2000;20(19):7345-52

47. Martin M, Dotti CG, Ledesma MD. Brain cholesterol in normal and pathological aging. Biochim. Biophys. Acta (BBA)-Mol. Cell Biol. of Lipids. 2010;1801(8):934-44.

48. Benvenuti S, Luciani P, Cellai I, Deledda C, Baglioni S, Saccardi R, et al. Thyroid hormones promote cell differentiation and up-regulate the expression of the seladin-1 gene in in vitro models of human neuronal precursors. J. Endocrinol. 2008;197(2):437-46.

49. Mokhtari T, Akbari M, Malek F, Kashani IR, Rastegar T, Noorbakhsh F, et al. Improvement of memory and learning by intracerebroventricular microinjection of T3 in rat model of ischemic brain stroke mediated by upregulation of BDNF and GDNF in CA1 hippocampal region. DARU J. Pharm. Sci. 2017;25(1):4

This work is licensed under a Creative Commons Attribution-NonCommercial 3.0 Unported License which allows users to read, copy, distribute and make derivative works for non-commercial purposes from the material, as long as the author of the original work is cited properly. 